\title{
Evolution of the Kuiper Belt during the Accretion of the Outer Planets
}

\author{
A. Brunini and M.D. Melita \\ Observatorio Astronómico - Universidad Nacional de La Plata \\ Paseo del Bosque S/N (B1900FWA) La Plata Argentina \\ CONICET melita@fcaglp.unlp.edu.ar
}

\begin{abstract}
A set of self-consistent simulations of the formation of Uranus and Neptune are performed to study the evolution of the native KBOs in the process. Our main goal is to have a deeper understanding of the impact of the formation of the outer planets on the present orbital structure of the trans-neptunian region. We aim to understand if resonance capture driven by the outward migration of Neptune can actually occur and its interplay with the invasion of massive planetesimals expelled from the Uranus-Neptune region as a byproduct of their formation. Also the putative present existence in the Oort reservoir of a population of objects originated in the Kuiper belt is analyzed.
\end{abstract}

\section{Introduction}

The Kuiper belt is a comet reservoir that extends beyond the orbit of Neptune and its actual outer limit is presently unknown. Recent studies of the formation of the outer Solar System indicate that proto-Neptune would have suffered a remarkable radial migration in the outwards direction (Fernández and Ip 1984, 1996). The outward radial migration would have enabled proto-Neptune to capture a great number of Kuiper belt objects (KBOs) into its exterior mean-motion resonances (MMRs) (Malhotra 1995). Also, as a byproduct of the formation of Uranus and Neptune, the trans-neptunian region would have been invaded by a number of big planetesimals of lunar size or more expelled in the process (Stern 1991).

Self-consistent simulations of the formation of Uranus and Neptune in the presence of an extended trans-neptunian disk have been performed to have a deeper understanding of the impact of the formation of the outer planets on the present orbital structure of the inner Kuiper belt region. We aim to understand if resonance capture driven by the outward migration of Neptune can actually occur and its interplay with the invasion of massive planetsimals expelled from the Uranus-Neptune region as a byproduct of their formation. Both scenarios have been proposed independently (Malhotra 1995, Morbidelli and Valsecchi 1997, Melita and Brunini 1999, Petit et al. 1999) and it is claimed that they play a major role in shaping the orbital distribution in the region.

\section{The numerical model}

We have based our model on the scenario proposed by Stevenson $(1982,1984)$. In this scenario, Uranus and Neptune accrete through successive pairwise collisions between large planetoids. 
Our numerical model consists of Jupiter and Saturn with their present masses and orbital parameters, 1000 massive planetesimals (proto-planets) distributed between 12 and $35 \mathrm{AU}$, each with a mass of $0.035 M_{\oplus}$, forming a total of $\sim 2 M_{\text {Neptune }}$. This protoplanet population corresponds to the objects that will accrete to form the two outermost major planets. Finally 500 test particles are set up with semi-major axes between 35 and $50 \mathrm{AU}$. The orbits of all objects are integrated using a second-order symplectic map and close encounters are treated specially using a Bulirsch-Stoer method. This is a new integrator scheme that will be described in detail in a forthcoming publication (Melita and Brunini 2000a).

A set of 12 runs have been performed, 3 of which resemble the present orbital structure of the Solar System. In the present article we base our discussion mainly on two of them, namely runs 2 and 5 .

Run 5
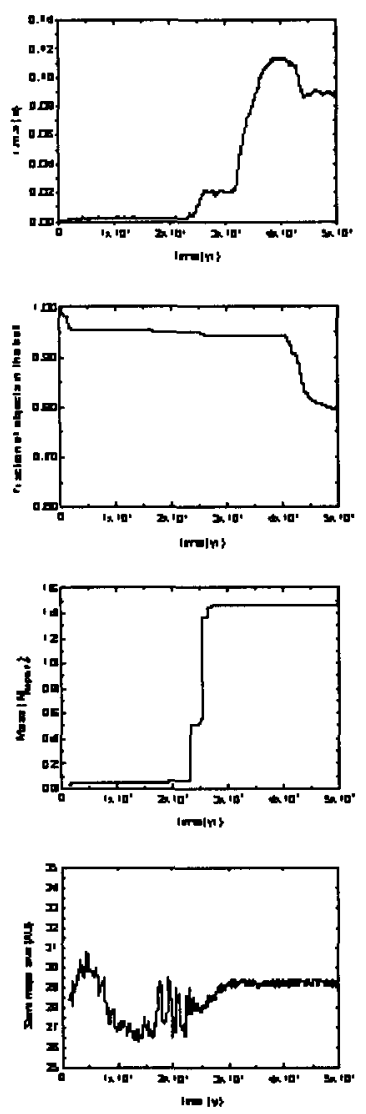

Run 2
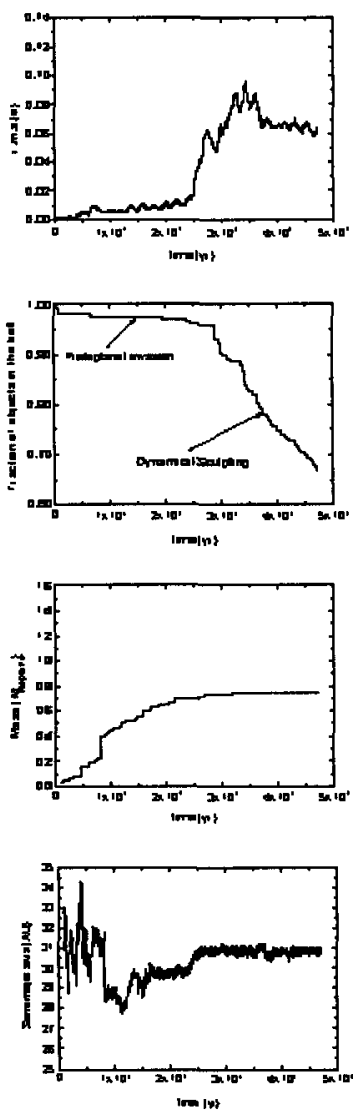

Figure 1. Mean eccentricity in the belt, fraction of objects remaining in the belt, semi-major axis and mass evolution of proto-Neptune for runs 2 and 5 . 


\section{The structure of the Kuiper belt right after the formation of Uranus and Neptune has been completed}

In Figure 1 the semi-major axis and mass evolution of proto-Uranus and protoNeptune is shown for both runs. As can be seen in the case of run 2, protoNeptune gets deeper into the belt. As a consequence, practically only very few KBOs can be found with semi-major axes below $40 \mathrm{AU}$ in this case (see Fig. 2), hence there is no sign of a resonant structure and, as evidenced by the characteristic shape of the final $a$ vs. $e$ graph, the excitation of the eccentricity appears to be dominated by the non-resonant perturbation of the planet. In the case of run 5 most of the lowest order mean-motion resonances fall into the belt, which causes excitation of the eccentricity of objects within them (see Fig. 2). The absence of objects in the inner portion of the belt in that case may also have been caused by a deeper penetration of proto-planets, which swept all the region (see Fig. 2). However most of the orbital excitation is due to the action of Neptune when this planet, having accreted most of its mass, has already settled into its final orbit (see Fig. 1).

\section{On resonance capture}

In general, resonance capture is possible when the orbits of two bodies are approaching one another as a consequence of the action of a dissipative force (Dermott et al. 1988). The transition from a non-resonant orbit to a resonant one depends, among other factors, on the speed at which the orbits are migrating due to the dissipation.

The final histograms of the number of objects in the belt do not show conspicuous evidence of resonance capture at the end of the simulations. However, for both simulations we have analyzed the number of objects in the corresponding resonant regions as a function of time, when there is a remarkable radial migration of proto-Neptune (already having accreted most of its mass). In both cases the mean eccentricity of the KBOs was below the critical limit, beyond which the probability of capture decays very rapidly (see for example Henrard 1981, Melita and Brunini 2000b). Although the capture efficiency is not as high as expected, probably due to the noise produced by encounters with surrounding objects (Melita and Brunini 2000b), temporary captures occur when the speed of migration falls within the theoretical limits. Table 1 shows the speed of the migration in those cases together with the corresponding limits that would make capture possible.

\section{Kuiper belt objects populating the Oort cloud}

In Table 2, we show the fraction of objects ejected from the belt by proto-Uranus and proto-Neptune as a function of their perihelion distance. These objects would have a certain probability to have end states in the Oort cloud, which can be estimated using the mean efficiency factors (as a function of perihelion distance) computed by Brunini and Fernández (2000). If we average the ejection values of runs 2 and 5 and estimate the initial mass present in the Kuiper belt to be between 10 and $30 M_{\oplus}$, then the primordial Kuiper belt would have contributed with 0.78 to $2.35 M_{\oplus}$ to the Oort reservoir. 

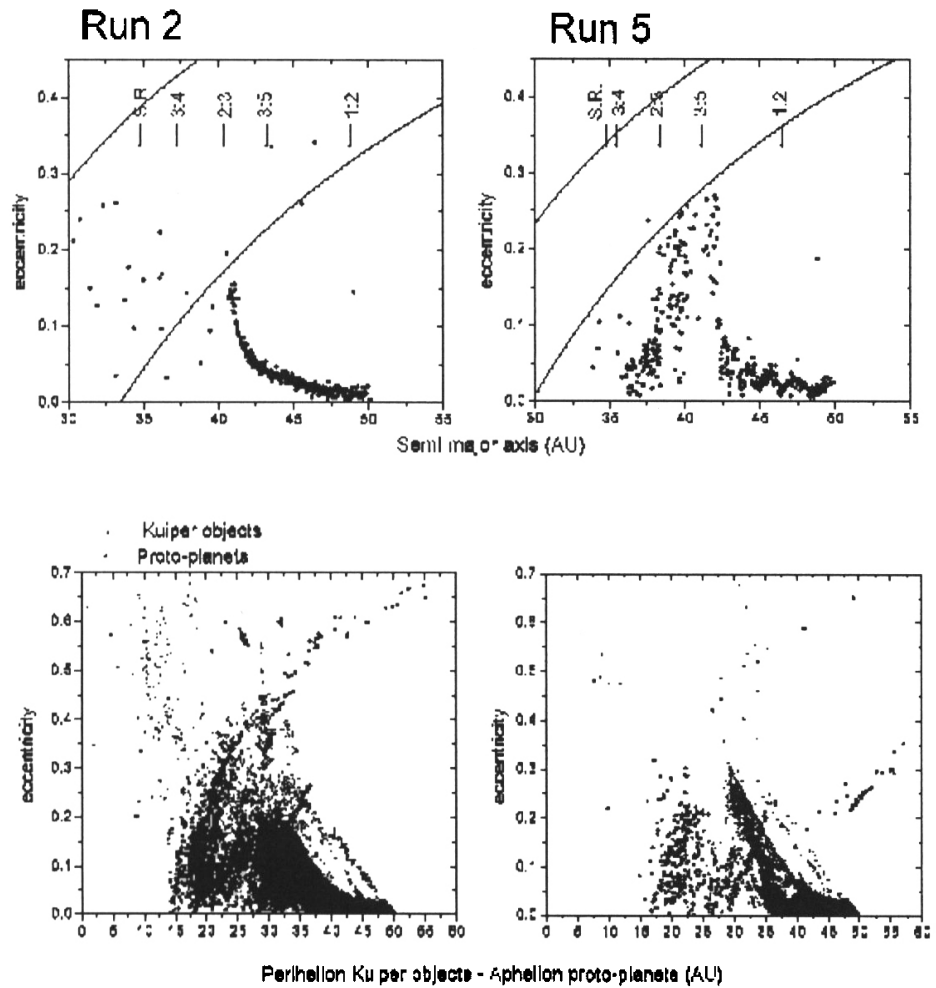

Figure 2. Final plots of the belt region for runs 2 and 5: a) eccentricity vs. semi-major axis; b) eccentricity vs. aphelion distance of proto-planets and perihelion distance of KBOs.

\section{Discussion}

From the simulations with end-states which resemble our planetary system, we can conclude that most of the dynamical sculpting of the inner Kuiper belt, including the notorious absence of objects with semi-major axes between $36 \mathrm{AU}$ and $39 \mathrm{AU}$ is due to the perturbations by Neptune. This last feature would also be a natural by-product of the resonance-sweeping scenario (Malhotra 1995). However, resonance capture does not appear to be a very conspicuous phenomenon though temporary captures are present in our experiments. In our models the initial mass distribution is not complete, since the contribution of the smaller planetesimals has not been taken into account. The interaction with the smaller objects would "smooth out" the evolution of the proto-planets and hence the resonant sweeping model cannot be rejected. Finally, it should be pointed out that, according to our estimations, a significant portion of the Oort cloud comets could have originated in the Kuiper belt.

Acknowledgments. We are grateful to IALP (CONICET) for their financial support. 
Table 1. Speed of the migration, $\tau$, taken from the numerical simulations, and the corresponding limit, $\tau_{\text {Lim }}$, that would allow capture.

\begin{tabular}{|l|cc|cc|}
\hline & \multicolumn{2}{|c|}{ Run 2 } & \multicolumn{2}{c|}{ Run 5 } \\
\hline$\tau\left(10^{-7} \mathrm{AU} / \mathrm{yr}\right)$ & 5.34 & 3.27 & \\
\hline & Res $2: 3$ & Res. 1:2 & Res 2:3 & Res. 1:2 \\
\hline$\tau_{\text {Lim }}\left(10^{-7} \mathrm{AU} / \mathrm{yr}\right)$ & $4.90(\mathrm{No})$ & 9.20 (Yes) & 22.5 (Yes) & 54.0 (Yes) \\
\hline
\end{tabular}

Table 2. Fraction of objects initially present in the Kuiper belt ejected from the system by proto-Uranus and proto-Neptune for each run.

\begin{tabular}{|c|c|c|}
\hline $\begin{array}{ll} & \text { Run } \\
\text { Perihelion } & \\
\text { Distance (AU) } & \end{array}$ & 2 & 5 \\
\hline $20-25$ & 0.028 & 0.044 \\
\hline $25-30$ & 0.11 & 0.084 \\
\hline $30-35$ & 0.114 & 0.03 \\
\hline $35-40$ & 0.008 & 0.03 \\
\hline$>40$ & 0. & 0.0 \\
\hline Total & 0.26 & 0.188 \\
\hline
\end{tabular}

\section{References}

Brunini, A. \& Fernández J.A. 2000, Icarus, 145, 580

Dermott, S.F., Malhotra, R. \& Murray, C.D. 1988, Icarus, 76, 295

Fernández, J.A. \& Ip, W.H. 1984, Icarus, 58, 109

Fernández, J.A. \& Ip, W.H. 1996, Planet. Space Sci., 44, 431

Henrard, J. 1981, Celest. Mech., 27, 3

Malhotra, R. 1995, AJ, 110, 420

Melita, M.D. \& Brunini, A. 1999, in Evolution and Source Regions of Asteroids and Comets, Proc. IAU Coll. 173, Astron. Inst. Slovak Acad. Sci., Tatranská Lomnica, Slovak Republic. (J. Svoreñ, E.M. Pittich and H. Rickman, Eds.), 37

Melita, M.D. \& Brunini, A. 2000a, Icarus, 147, 231

Melita, M.D. \& Brunini, A. 2000b, Icarus, 147, 205

Morbidelli, A. \& Valsecchi, G.B. 1997, Icarus, 128, 464

Petit, J.M., Morbidelli, A. \& Valsecchi, G.B. 1999, Icarus, 141, 367

Stern, S.A. 1991, Icarus, 90, 271

Stevenson, D.J. 1982, Planet. Space Sci. 30, 755

Stevenson, D.J. 1984, Lunar Planet. Sci. XV, 822 